\title{
Strategy and Automation of the Quality Assurance Testing of MaPMTs for the LHCb RICH Upgrade
}

\author{
Konstantin Gizdov ${ }^{\mathrm{a}, 1, *}$, on behalf of the LHCb RICH Collaboration \\ ${ }^{a}$ School of Physics and Astronomy, University of Edinburgh, James Clerk Maxwell Building, Peter Guthrie Tait Road, Edinburgh, EH9 \\ 3FD, United Kingdom
}

\begin{abstract}
The LHCb RICH system will undergo major modifications for the LHCb Upgrade during the Long Shutdown 2 of the LHC, and the current photon detectors will be replaced by Multi Anode PMTs. The operating conditions of the upgraded experiment puts forth significant requirements onto the MaPMTs in terms of their performance, durability \& reliability. Presented is an overview of the testing facilities designed and used to vet 3100 units of Hamamatsu R13742 and 450 units of Hamamatsu R13743 during the short 2 year testing period. Discussed are the hardware architecture, the different read-out, power and control components, as well as the novel extensible software framework to steer the procedure. Finally, the operation of four automated stations, that have been deployed in two separate labs, is reported, with each station capable of fully characterising 16 MaMPTs per day.
\end{abstract}

Keywords: RICH Upgrade, MaPMT, PDQA, Automation, Microservice, Finite State Machine

\section{Introduction}

The LHCb RICH detector will be upgraded during 3 the LHCb Upgrade 1a to satisfy the new operating re- 28 quirements. The luminosity will increase to $\mathcal{L}=2 \times 29$ $10^{33} \mathrm{~cm}^{-2} \mathrm{~s}^{-1}$ and thus the read out rate will increase from ${ }_{30}$ $1 \mathrm{MHz}$ to $40 \mathrm{MHz}$. Consequently, the current Hybrid Photon Detectors (HPDs) will be replaced with Multi Anode 32 Photomultiplier Tubes (MaPMTs) with external read out electronics [2]. The entire upstream RICH detector (RICH 1) will be equipped with Hamamatsu ${ }^{2}$ R13742 MaPMT (64 channels, a custom modification to the R11265). The central high occupancy area of the downstream RICH detector (RICH 2) will be equipped with the R13742 as well, while the low occupancy regions requiring lower granularity will be equipped with the Hamamatsu R13743 (64 channels, a custom modification to the H12699). As a result 3100 units of R13742 and 450 units of R13743 will be used in the RICH system (including spares). Ensuring the operation qualities of the new MaPMTs is the Photon Detector Quality Assurance program. It extended over two 1 years (2016-2018) with the main aim of characterising the units, assuring minimum specifications, pre-selecting tubes ${ }_{33}$ with similar characteristics to be installed in functionally ${ }_{34}$ related areas of the detector and gather initial calibration 35 variables.

\footnotetext{
* Corresponding author

Email address: k.gizdov@ed.ac.uk (Konstantin Gizdov)

${ }^{1}$ University of Edinburgh

${ }^{2}$ Hammamatsu Photonics: http://www.hamamatsu.com
}

\section{Automation of the Quality Assurance Testing of MaPMTs}

\subsection{The Photon Detector Quality Assurance (PDQA) and Test Bench}

The LHCb RICH PDQA program was carried out in two sites (Edinburgh, UK and Padova, Italy) equipped with test stations each and requiring a high level of reliability, reproducibility and consistency. A custom read

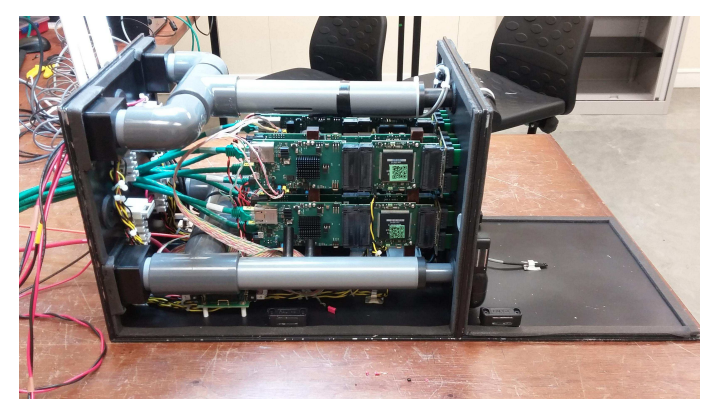

Figure 1: Test box with fully integrated Front-End readout, data acquisition, cooling and environment monitoring.

out chain (Fig. 1) has been developed in order to characterise the MaPMTs. The MAROC3, developed by Omega Microelectronic $3^{3}$ has been selected as the front-end chip, integrating and digitising the charge pulses of $64 \mathrm{MaPMT}$ channels in parallel. The signal spectra allow the extraction of MaPMT gain, signal loss and the dark count rate,

$\sqrt[3]{\text { omega.in2p3.fr }}$ 
qualifying the single photon-detection capability of each 93 channel.

A compact test box has been designed and produced in order to assess several MaPMTs in controlled and reproducible conditions (Fig. 1). The test box comprises of two compartments separated by a light-tight wall. The back compartment houses the front-end and the digital readout. The front area contains the voltage divider chain and the MaPMTs. It is important to monitor and control the temperature of the environment which affects the MaPMTs performance [2. The temperature inside the box is controlled by forced-air cooling.

The light source consists of two pulsed LED (470 nm) coupled with two optical fibres; the light from the two fibres is directed on a mirror mounted inside on the box cover and then reflected on the photocathode plane providing uniform illumination.

A custom slow control based on the Aria-G25 FPGA 4 has been produced in order to control and monitor the $\mathrm{HV}$, light intensity and to monitor the temperature and ${ }_{95}$ humidity sensors.

The setup is designed to test $16 \times \mathrm{R} 13742$ or $4 \times 97$ R13743. The PDQA procedure aims to verify the spec- 98 ification provided by Hamamatsu: the dark count rate 99 $(<16 \mathrm{kHz})$, the average gain $(>1 \mathrm{Me})$, the gain unifor-100 mity and the peak-to-valley ratio $(\mathrm{P} / \mathrm{V})$ for single photons.101 Long periods of operation in the $\mathrm{LHCb}$ environment will ${ }_{102}$ degrade the MaPMT performance over the years. This re-103 sults in a gradual loss of gain, which can be compensated ${ }_{104}$ by adjusting the high voltage (HV) supply. Therefore the 105 signal gain response of the MaPMT as a function of the ${ }_{106}$ $\mathrm{HV}$ is a crucial part of the PDQA procedure in order to de-107 termine the minimum HV setting to achieve single photon detection. The test protocol for the production has been established as: dark counts settling overnight at nominal $\mathrm{HV}=1000 \mathrm{~V}$, burn in test at $\mathrm{HV}=1100 \mathrm{~V}$, data collection at different HV values: $1100 \mathrm{~V} \rightarrow 850 \mathrm{~V}$. The number of measurements performed on the many devices during the two years of PDQA required a high level of automation of the test facilities. Thus, control software had to be developed to carry out the test program with minimal requirement for operator intervention - a robust system for handling data, fitting spectra and to speed up the data interpretation and the measurement validation.

\subsection{Microservices Architecture and System Automation}

The full PDQA test station consists of the test box, LED driver, slow control, power supplies and a workstation computer. In order to satisfy the requirement for ${ }^{108}$ reliability, reproducibility \& consistency the control soft- ${ }^{109}$ ware needs to be able to operate the test station fully and ${ }^{110}$ autonomously for long periods of time. It must be able ${ }^{111}$ to configure and execute each test and complete the pro- ${ }^{112}$ cedure without the need for human operator intervention. ${ }^{113}$

$\sqrt[4]{\text { http://www.acmesystems.it/aria }}$
This in itself presents a further requirement - namely, it needs to be able to handle errors, recover from them and continue operating.

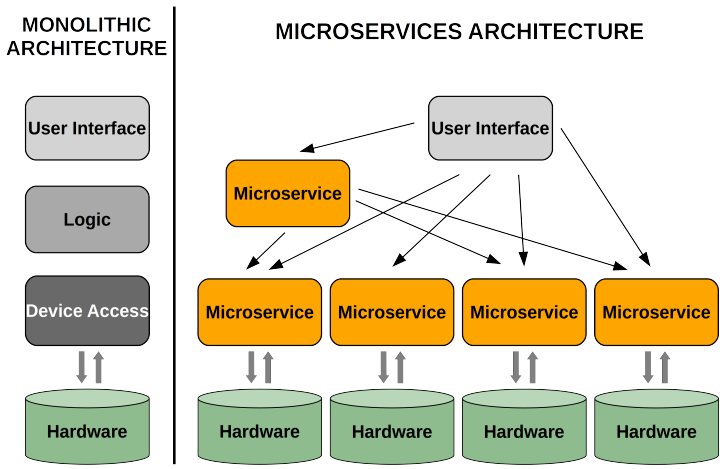

Figure 2: Model representation of microservices architecture compared to a monolithic architecture.

Considering the complexity of the whole system, in terms of devices, their configuration, interaction and the resulting intricate logic, the control software design presents a significant hurdle. In conventional monolithic type software (Fig. 2) there is a single clear hierarchy of execution, logic and control. This presents a problem when an increasing amount of different concurrent events needs to be handled. Having a single logic core means the overall complexity grows and allows for accumulation of mishandled edge cases and undefined behaviour. The most crucial concern here, however, remains that monolithic applications cannot easily recover their state from a fatal fault and continue the testing procedure.

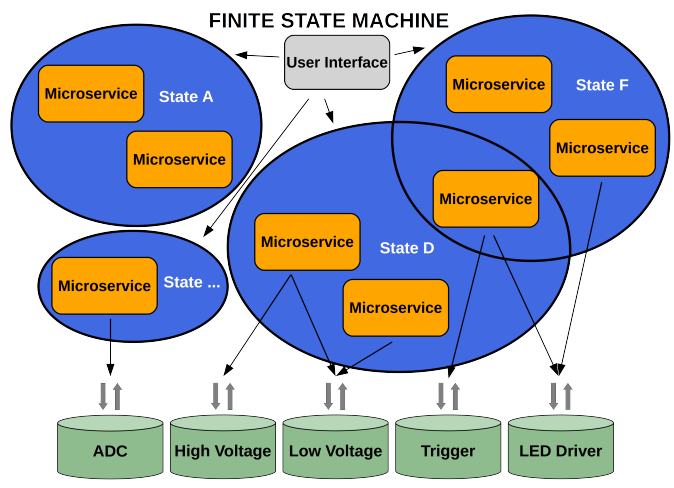

Figure 3: Structure of the finite state machine automating the PDQA testing procedure.

Thus, it was decided to adopt a modern approach of designing the control software and utilize the Microservices architecture 4. Microservices are smaller independent logical units that carry simpler responsibilities than conventional applications. Each microservice executes on its own, has its own logic and all of them are linked by a common communication protocol (Fig. 2). A microservice, thus provides a 'service' to the rest of the connected units 
and other microservices can request that service. For example, a service controlling the high voltage executes on its own, keeps track only of the $\mathrm{HV}$ and answers to requests such as reporting the $\mathrm{HV}$ status or setting an $\mathrm{HV}$ to a value. It only needs to know the HV software driver and how to respond to requests.

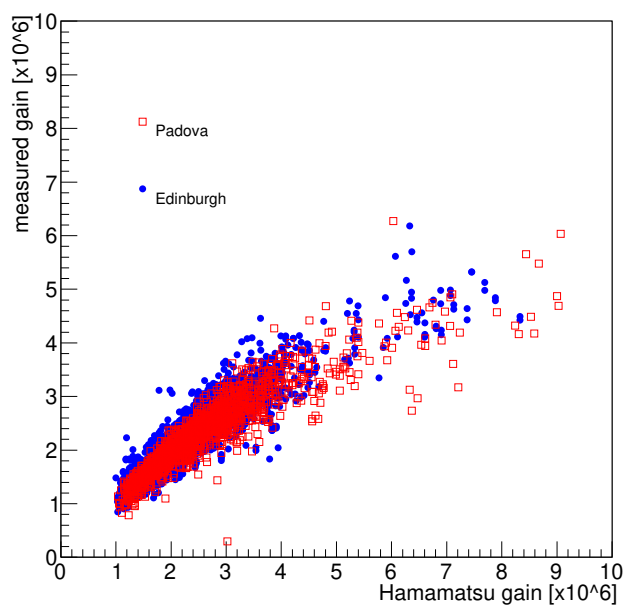

Figure 4: Comparing the R13742 (1 in) PMT gain between the Hamamatsu datasheet and the result from the PDQA testing procedure.

In order to facilitate the operation of more compli- ${ }^{153}$ cated actions, there can be another microservice unit that ${ }^{154}$ 'knows' when to switch on the HV and to what value. $\mathrm{So}^{155}$ it can carry the logic of HV safety and which kind of test is ${ }^{156}$ currently being carried out. It does not need to know how ${ }^{157}$ to control the HV, just how to request some other unit ${ }^{158}$ to do it. Taking this concept further allows for building ${ }^{159}$ a structure or network of units capable of executing very ${ }^{160}$ complicated tasks, while designing and operating each of ${ }^{161}$ them is much simpler. Moreover, it grants multi-threaded ${ }^{162}$ operation inherently as part of the design without extra ${ }^{163}$ effort. Finally, it addresses the main concern of contin ${ }^{-164}$ ued operation and recovery - microservices do not store ${ }^{165}$ the whole state of the system. Thus each can fail and ${ }^{166}$ be restarted when needed by the rest of the units with ${ }^{167}$ virtually no loss of information. The final control software builds on this premise (Fig 3). It implements a finite ${ }_{168}$ state machine (FSM) with a graphical user interface (GUI) where each logical state is operated by a microservice. $\log _{-}{ }_{-169}$ ical state microservices rely on other microservices to ac- ${ }^{170}$ complish their task as they are only aware of the high-level ${ }^{171}$ logic. The GUI is also implemented as several microser- ${ }^{172}$ vices. Thus, the software implements a general application ${ }^{173}$ programming interface (API) for communication with and $d^{174}$ in-between the microservices. It uses the $\mathrm{C}++^{5}$ language ${ }^{175}$ for low-level \& high-performance units, which the GUI ${ }^{176}$

\footnotetext{
${ }^{5}$ https://isocpp.org/
}

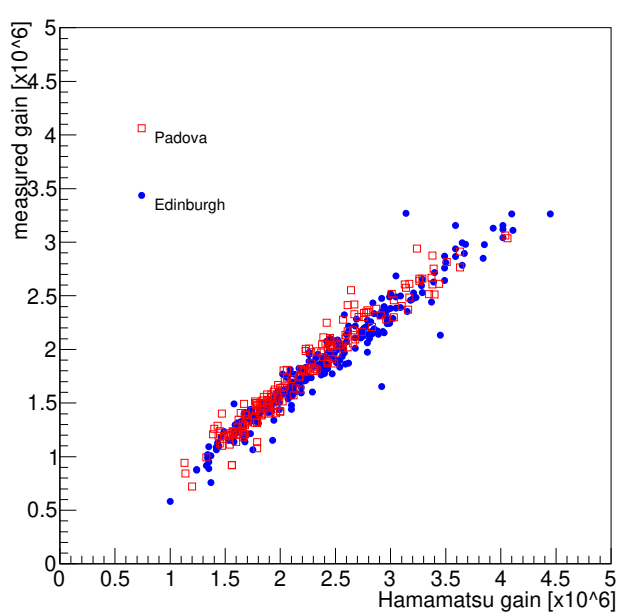

Figure 5: Comparing the R13743 (2 in) PMT gain between the Hamamatsu datasheet and the result from the PDQA testing procedure.

units are implemented in LabVIEW $W^{6}$ ROOT 7 is used for data analysis and $X M L^{8}$ for human readable settings and logs.

\section{Results}

The PDQA Automation [5] software was deployed in two labs and four testing stations in total and performed as expected. Each station was able to operate with minimal human intervention (only for mounting the MaPMTs and starting the procedure) for the duration of the tests, handling fully the procedure, data taking and interpretation. The first requirement that the MaPMTs must satisfy is a minimum average gain of $1 \mathrm{Me}$. Results obtained for the R13742 \& R13743 are shown in Fig. 4 \& 5 respectively, where the extracted average gain is compared to the value provided by Hamamatsu. There is broad agreement between the gain provided by the company and that measured in our labs, taking into account the different measuring methods used. The requirement that each tube has a gain of at least $1 \mathrm{Me}$ is clearly satisfied.

\section{Conclusion}

During the Quality Assurance, the automated system tested a total number of $3100 \& 450$ MaPMTs. The system was deployed on four stations in two labs and consistently characterised $16 \times 1$ in $(4 \times 2$ in $)$ PMTs per station per day. The results show excellent gain, uniformity and single photon resolution. Moreover, the automation allowed for thorough consistency between the two labs and overall efficiency increase.

\footnotetext{
${ }^{6}$ https://www.ni.com/labview

${ }^{7}$ https://root.cern.ch/

${ }^{8}$ https://www.w3.org/TR/REC-xml/
} 


\section{References}

[1] LHCb Collaboration, LHCb PID Upgrade Technical Design Report, CERN-LHCC-2013-022, LHCB-TDR-014

[2] L. Cadamuro et al., Characterization of the Hamamatsu R11265-103-M64 multi-anode photomultiplier tube, JINST 9, P06021 (2014) doi:10.1088/1748-0221/9/06/P06021 arXiv: 1403.3215

184 [3] S. Gambetta [LHCb RICH Collaboration], First results from 185 quality assurance testing of MaPMTs for the LHCb RICH up186 grade, Nucl. Instrum. Meth. A 876, 206 (2017). doi:10.1016/ 187 j.nima.2017.02.079

188 [4] Lewis, J., Fowler, M., 2012. Microservices [WWW Doc189 ument].martinfowler.com. https://martinfowler.com/ $190 \quad$ articles/microservices.html (accessed 10.9.18)

191 [5] Gizdov, K., Gallorini, S., 2018. PDQA Automation. https:// 192 gitlab.cern.ch/kgizdov/pdqa-automation 